\title{
AN ENHANCEMENT OF RTS/CTS CONTROL HANDSHAKE IN CSMA/CA BASED MAC PROTOCOL FOR AN EFFICIENT PACKET DELIVERY OVER MULTIHOP WIRELESS MESH NETWORK (WMN)
}

\author{
Vigneswara Rao Gannapathy ${ }^{1}$, Ahamed Fayeez Bin Tuani Ibrahim², \\ Zahriladha Bin Zakaria ${ }^{3}$, Abdul Rani Bin Othman ${ }^{4}$, Anas Abdul Latiff ${ }^{5}$ \\ 1, 2, 3, 4, 5 Lecturer, Department of Electronics and Computer Engineering, Universiti Teknikal Malaysia Melaka, Melaka \\ (UTeM), Malaysia,vigneswara@utem.edu.my,fayeez@utem.edu.my,zahriladha@utem.edu.my,rani@utem.edu.my, \\ anasabdullatiff@utem.edu.my
}

\begin{abstract}
The Carrier Sense Multiple Access with Collision Avoidance (CSMA/CA) based Medium Access Control (MAC) protocol uses a short request-to-send (RTS) and clear-to-send (CTS) control packets prior of actual data packet transmission. The function of the CSMA/CA based MAC protocol in wireless communication is to facilitate wireless nodes (i.e. laptop, PC, smart phones and etc) to access a wireless medium efficiently. Besides, it is also efficiently manage the wireless medium when more wireless nodes involving in transmission activity in the network. This protocol allows all wireless nodes in the network to communicate between each other without collision. Since collisions may only occurred on the RTS control packets thus it can reduce the actual data collision on the medium. The RTS/CTS control handshake provides better performance and reduce the duration of collision especially when long data packets are transmitted over wireless medium. However, even though the CSMA/CA based MAC protocol working effectively and provides better throughput performance over single hop communication but it performance degrade significantly when directly applied in multihop Wireless Mesh Network (WMN). The reason behind this poor performance is due to the inefficient packet delivery of CSMA/CA based MAC protocol in multihop network. The exchange of RTS and CTS signaling that required at each hop throughout the multihop network eventually will cause the large overheads and subsequently degrade network throughput. Therefore, in this paper, an enhancement of RTS/CTS handshake has been proposed in order reduces the signaling overheads and ultimately allows a smooth packet delivery in the multihop network without any collision. In this work, the multihop network performances are evaluated analytically in terms of throughput and overhead. It is proven that the new method of RTS/CTS handshake provides significant improvement in throughput and overhead.
\end{abstract}

Keywords: Medium Access Control (MAC) protocol; Signaling; Overheads; Multihop communication

\section{INTRODUCTION}

Wireless communication between network nodes has become more popular in recent years and has been integrated into people's daily life, e.g. wireless Internet connection, hands free and etc. Wireless Mesh Network (WMN) is a wireless communication network that consists of network nodes connected in mesh-style topology. It become an emerging technology and plays an important role in the next generation wireless communication. WMN architecture is built based on the principles of multihop communication with addition of mesh topology to link all the nodes inside the network [1]. Due to the low cost, ease of deployment and regardless of physical layer (PHY) being used, WMN can provides wide network coverage with seamless roaming, the internet connection to remote and rural areas and solving blind spot problem for the existing wireless and mobile communication networks such as WiMAX and $3 \mathrm{G}$ networks. This type of infrastructure is considered relatively stable, can provide reliable services and fairly inexpensive.

A WMN is characterized by self-organizing, self-configuring and self-healing capabilities, with the mesh nodes in the network automatically establishing and maintaining mesh connectivity among themselves [2]. Typical WMN architecture consists of mesh routers (MRs), mesh clients (MCs) and single or multiple Internet gateways (IGWs) as shown in Figure 1. The MRs acting as bridges in order to forward packets until it reach their destination [3]. The MRs having minimal mobility are wireless nodes linked to one another to extend the coverage area and formed the backbone whereas the MCs can be connected to MRs in traditional way and can roam easily. If a MC needs to communicate with another node that is not within its communication range, it forwards the packet to its nearest MR. The packet is then 
forwarded over the MR backbone according to routing protocol, until it reaches a MR that can forward the packet to the destination node.

One of the major advantages of WMN lies on fault tolerance capability against network failures. Unlike other wireless networks, failure of a single node (i.e base station or access point) leading to unavailability of communication services over a large geographical area. The WMN provide high fault tolerance even when a number of MRs fail [1]. If some MR nodes in the network are down, the forwarded traffic can be delivered to the destination via some other intermediate MR nodes. This implies that the WMN provides the network with higher fault tolerance to the failures of MR nodes and links.

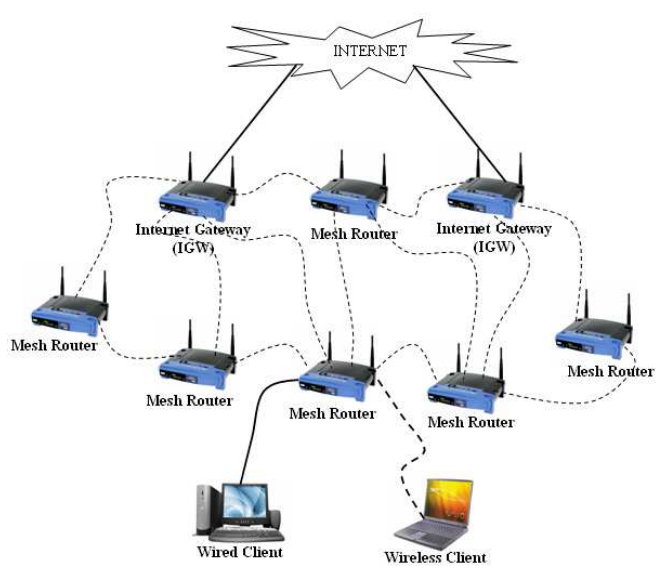

Fig1: Wireless Mesh Network Architecture

Medium Access Control (MAC) protocols employed in multihop WMNs to resolve contentions for accessing the shared medium which is encountered many issues especially exposed node problems in multihop communications [4]. Apart from that as the number of hops in WMNs increases, the optimal overall throughput of existing MAC protocol and its derivatives are not achievable. So, the enhancements of the existing IEEE 802.11 Distributed Coordination Function (DCF) MAC protocol have been proposed in this work. Since the source node in WMN relaying its data packets over multiple hops until reach destination node, the large number of overhead due to signaling at each intermediate hop will degrade the throughput significantly.

In this paper, we are focusing on how to reduce the large signaling overhead problems in multihop networks. This can be done by reducing the number of signaling packets needed at each intermediate hops until the data packet reaches its destination in multihop WMNs. This approach is expected to reduce the signaling overheads thus improve the performance significantly.

\section{PROBLEM STATEMENT}

One of the reasons behind throughput degradation in multihop communication is number of signaling packet that required when transmitting a data packet from one node to destination via multiple intermediate nodes. As shown in Figure 2, let's consider source node A has data packet to be transmitted to destination node E. Since node E does not include node A's transmission range, node $\mathrm{A}$ has to transfer its data packet via some intermediate to reach its destination. In this case, the data packet must go through three intermediate nodes, which are node $\mathrm{B}$, node $\mathrm{C}$ and node $\mathrm{D}$ in order to reach the destination. When the existing MAC protocol employ in this type of multihop communication, the four way signaling handshake (i.e RTS/CTS/ACK/DATA) will be performed at every each intermediate nodes thus increasing the delay and reduce throughput. This is the nature of IEEE 802.11 MAC protocol that is initially designed for single hop transmission in mind. However, this approach degrades the throughput of WMN significantly.

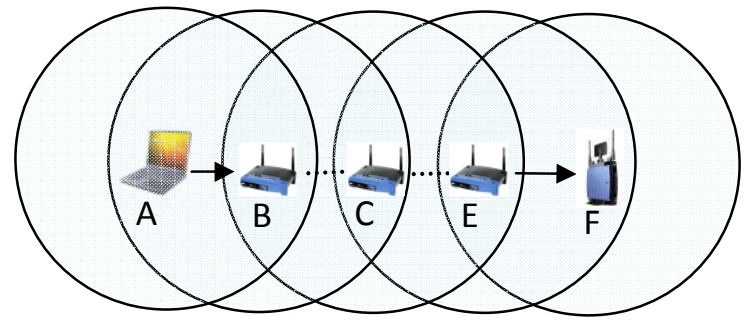

Fig2: Multihop Transmission via intermediate nodes

The amount of signaling packets is needed to transmit a single data packet in multihop network increase proportionally with number of hops. The latency caused by signaling overhead will drop the throughput significantly. A smooth and efficient data forwarding operation is require in order to improve the throughput in multihop WMN. This will reduce the signaling overhead thus improve the throughput accordingly. Therefore, an enhanced MAC protocol which is efficiently forward the data packet is introduced and presented in this paper. In our approach the RTS and CTS signaling exchange is only occurred at first hop when the node contending for the shared channel. Whereas at the subsequent hops, it will initiates its transmission according to the proposed technique and reduce the signaling overheads.

\section{PROTOCOL DESCRIPTION}

The novelty of the proposed protocol is its efficiency in enabling the forwarding operation in collision free manner. This approach reduces the amount of signaling overhead that needed when the nodes relay the data packet to the nearest gateway in multihop fashion. 
In this paper, we have proposed a simple enhancement to existing 802.11 MAC to reduce the amount of signaling packet that requires at each intermediate node. Since every each node in the network has the omnidirectional characteristic, thus they can overhear the transmission activity which is going on around them. This advantage is used to convey the data packet to subsequent nodes.

The Figure 3 illustrated the forwarding operation of proposed protocol. As shown in this figure, the data packet from client A has to be relayed via some intermediate nodes until it reaches its nearest internet gateway (i.e. node $n$ ). According to proposed protocol, the mRTS and mCTS signaling exchange only will be performed at first hop when the source node initiates the transmission. This exchange is requires to get an access to the channel. Upon successful transmission at first hop, the data packet will be relayed through subsequent relaying nodes to IGW1 without initiating the mRTS control packet. Therefore the mRTS control packet that needed at each intermediate relaying node can be suppressed accordingly.

Let assume node A will broadcast the mRTS control packet to start its transmission to node B. Next, node B will permit this transmission by responding the mCTS control packet back to it. Due to the overhearing capability, each of the neighboring nodes in the vicinity of node B (i.e. node C) will overhear this mCTS control packet. In order to identify the next relaying node, the RID mechanism is applied in this protocol. According to the MAC addresses and RID that contain in mCTS control packet, node $\mathrm{C}$ is able to recognize the next relaying node for that respective data packet. Thus, once node C overhears mCTS control packet, it will activate the timer to send the invitation and asked for the data packet to be forwarded (will explain in next paragraph). The timer will be activated to be equal to the time taken by node $\mathrm{B}$ to receive the DATA packet from its upstream node and until it transmits mACK packet. All the neighboring nodes (in the vicinity of the node B) will be notified on upcoming forwarding operation through the mACK packet which is sent by node B at the end of its transmission.

The nodes which overhear the mACK packet merely increase the duration of its NAV based on the Duration/ID field in that mACK packet to reserve the channel for subsequent forwarding operation. The increment duration will indicate for how long the nodes should further defer the transmissions on the channel. This NAV increment also performs by the target of the mACK (i.e. upstream node, node A). Thus, once node $\mathrm{A}$ received the $\mathrm{mACK}$ packet from node $\mathrm{B}$, so it will increase its NAV duration and will remain silent until entire forwarding process (from node B to node C) completes. It will be set, to be equal to time taken for the transmission of mCTS, DATA, and mACK packets, plus three SIFS intervals. Based on RID information in the mACK packet, the node $\mathrm{C}$ will aware that the corresponding mACK packet is from its upstream node and will refrain from increasing its NAV duration, thus continue decreasing its timer.

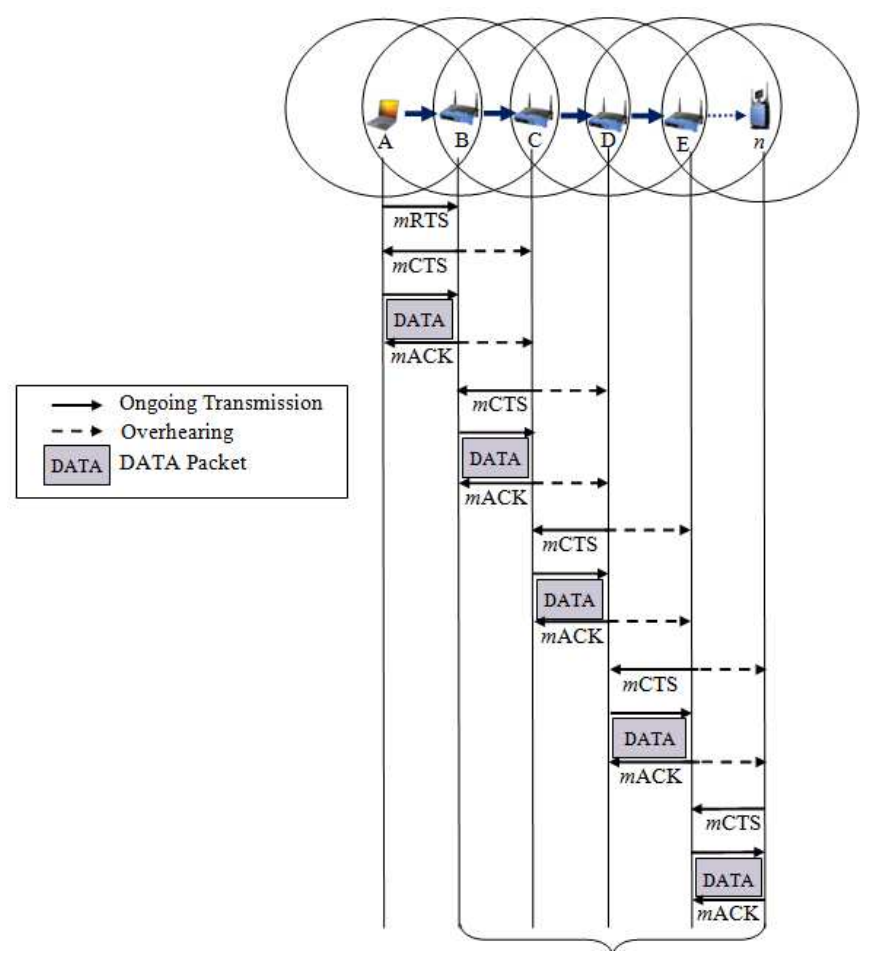

Fig3: Efficient forwarding operation

Upon timeout, the node $\mathrm{C}$ will broadcast its mCTS control packet to send the invitation to the node $\mathrm{B}$ to forward the data packet. All other nodes which overhear the mCTS control packet (the nodes in the vicinity of node $\mathrm{C}$ ) will updates their respective NAV and will remain until the entire forwarding process completes. Similarly, the next corresponding relaying node (i.e. node D) will overhear this mCTS control packet sent by node $\mathrm{C}$ and will activate its timer accordingly. The timer will be activated to be equal to the time taken by node $\mathrm{C}$ to receive the data packet from its upstream node and until it transmits mACK packet. Upon the timer expires, node D will send the mCTS control packet to invites the node $\mathrm{C}$ to forward the data packet. This mechanism continues to repeat at each intermediate relaying nodes until it reach the gateways (i.e. $n$ ). Thus, the mRTS packet that suppose sent by sender node can be suppressed.

\section{NETWORK MODEL}

The network we considered consists of $n$ mesh routers, mesh clients and gateways. Gateways to the Internet are chosen from a set of $\mathrm{n}$ mesh routers. The other mesh routers are referred as intermediate mesh routers which is expanding the network coverage and providing reliable links to gateways. 
The network topology is shown in Figure 1. Each MR is equipped with single interface except the gateway (another interface to Internet) and has a common transmission range, $r$. Both the mesh clients and mesh routers use the same physical layer (PHY) frequency band by which in this work we consider the use of IEEE 802.11 PHY. The transmission rate is constant and packets are forwarded in a multihop fashion to the gateway. For ease of explanation and without loss of generality, we consider unidirectional traffic, i.e., traffic only going from mesh nodes to the gateway. We assume that each mesh router has a fixed transmission rate at $54 \mathrm{Mbps}$ and range of 100 meters. Thus only two routers can set up a link (i.e within communication range) and communicate between them whenever possible. As for the mesh clients, some of them are associated to a certain mesh router forming a cell.

\section{PERFORMANCE RESULT}

Figure 4 and 5 depicts the variation of delay and throughput as a function of number of hops respectively. It can be observed from both results that the proposed protocol outperforms IEEE 802.11 MAC protocol. As depicts in figure 4, the delay which is consumed by the proposed protocol to transmit the data packet from source to destination is $7.37 \mathrm{~ms}$, meanwhile the delay consumed by the existing protocol to transmit its data packet from source to destination is $8.27 \mathrm{~ms}$. This is shows that the proposed protocol can reduce the delay approximately $10.88 \%$ when it transmit its data packet through 10 hops.

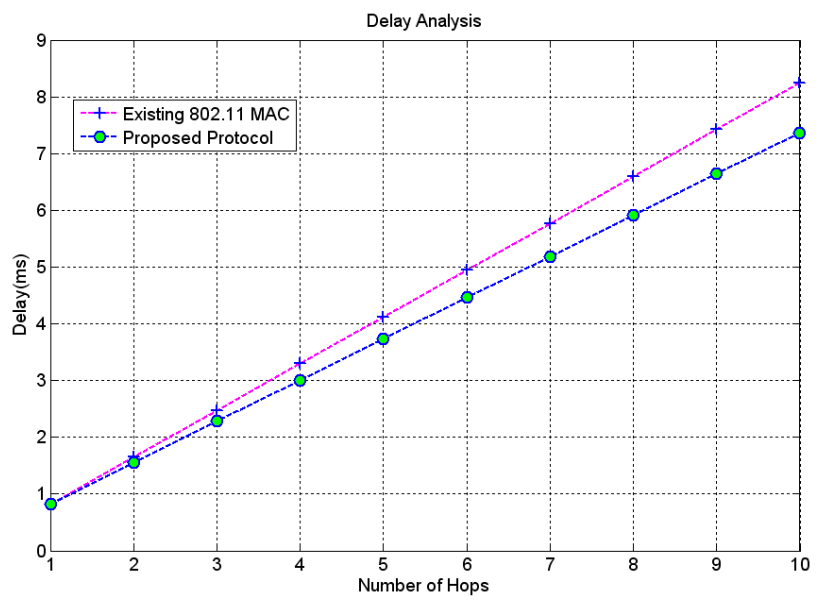

Fig4: Delay analysis

Moreover as depicts in figure 5, the throughput which is achieved by the proposed protocol in order to transmit the data packet through 10 hops is 2.9 Mbps, meanwhile the throughput achieved by existing protocol is only $2.1 \mathrm{Mbps}$. This is shows that the proposed protocol can improve the throughput approximately $38.1 \%$. Since the existing IEEE 802.11 MAC was designed with one hop communication in mind, it suffers throughput degradation when applied for multihop topology as shown in Figure 3. This observation is due to the smaller delay per payload which is experienced by proposed protocol. Moreover the proposed protocol outperforms the existing when number of hops increasing as shown in Figure 4 and 5. Obviously this observation shows that the proposed protocol reduced the delay caused by signaling overhead.

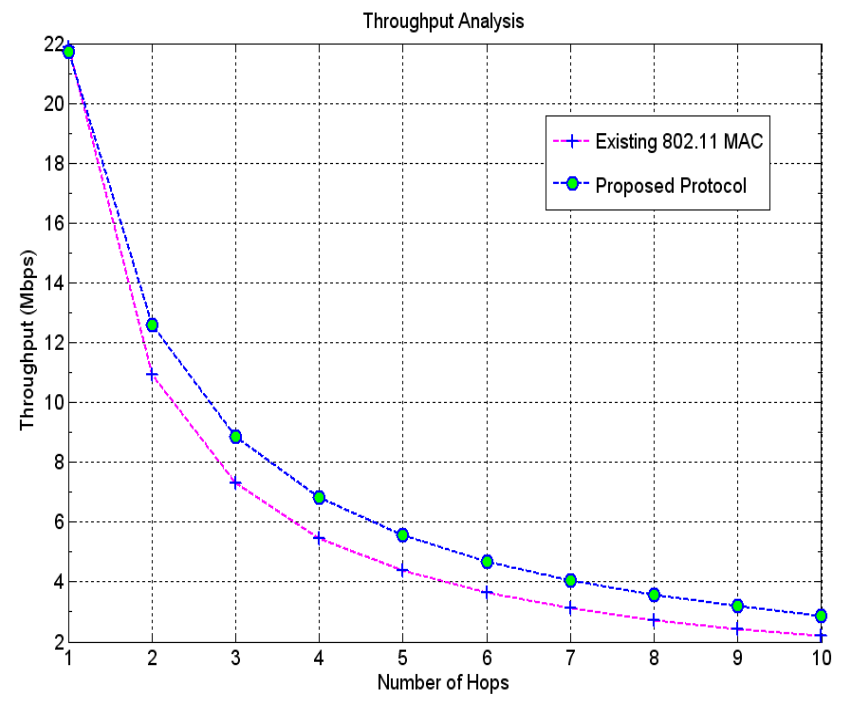

Fig5: Throughput analysis

\section{CONCLUSIONS}

By presenting the problem encountered in an IEEE 802.11based multihop network, it can be concluded the current version of this MAC protocol does not function well in multihop WMNs. In order to overcome the problem, we have proposed an efficient forwarding operation to forward the data packet with a fewer signaling overhead. Therefore, a set of enhancement to the existing IEEE 802.11 DCF MAC was introduced to reduce the amount of signaling overhead required at every hop until the data packet reaches its destination. The analytical models were developed and the multihop WMN performances are evaluated in terms of throughput and delay. It proven that proposed protocol outperforms the existing IEEE MAC protocol in throughput of multihop WMN.

\section{ACKNOWLEDGEMENTS}

The authors would like to take this opportunity to thanks those who are contributes directly or indirectly in completion of this article and also for their constructive comments. In addition, the authors also would like to express our gratitude to Universiti Teknikal Malaysia Melaka (UTeM) for the support and encouragement. The work in this paper was funded by short term grant (PJP/2011/FKEKK(40B)/S00972) that offered by UTeM. 


\section{REFERENCES}

[1] Zhang, Y., Luo, J., \& Hu, H. (2007). Wireless Mesh Networking - Architectures, Protocols and Standards, 1st ed., New York: Taylor \& Francis Group.

[2] Akyildiz, I. F., Wang, X., and Wang, W. (2005). Wireless Mesh Networks: A Survey. Computer Networks Journal, vol. 47, pp. 445-487.

[3] Siddiqui, M.S. \& Hong, C.S. (2007). Security Issues in Wireless Mesh Networks. International Conference on Multimedia and Ubiquitous Engineering (MUE'07), Computer Society.

[4] Akyildiz, I.F. \& Wang, X. (2005). A Survey On Wireless Mesh Networks. IEEE Communications Magazine, Vol. 43, pp. 23-30.

[5] Toh, C.K., Vassiliou, V., Guichal, G., \& Shih, C.H. (2000). MARCH: A Medium Access Control Protocol for Multihop Wireless Ad Hoc Networks. Proceedings of IEEE Military Communication, pp. 512 - 516.

[6] Vigneswara Rao, Gannapathy., Suaidi, Mohamad Kadim., Johal, Muhammad Syahrir Bin., Chuan, Lim Kim., Ramli, Nordin., Mohamad, Hafizal. (2010). “A Smooth Forwarding Operation in Wireless Mesh Network," in IEEE 10th Malaysia International Conference on Communications (MICC), pp. 83-87.

[7] Zakaria, M.S., Ahma, Suaidi, M.K., Johal, M.S. (2010). Modeling and Performance Analysis of Throughput Received Power Relationship for Indoor Wireless Mesh Network (WMN). Indonesia - Malaysia Microwave Antenna Conference (IMMAC 2010). Universitas Indonesia, Depok, Indonesia.

\section{BIOGRAPHIES}

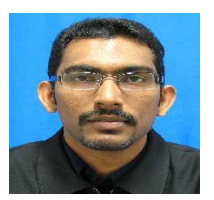

Engr. Vigneswara Rao Gannapathy currently serving as a lecturer in Universiti Teknikal Malaysia Melaka (UTeM) and he actively involves in research activities which is related to electronics and wireless networking. His research direction has focused on Wireless Mesh Networks which emerged as a key technology for next-generation wireless networking.

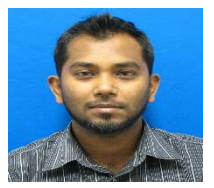

Ahamed Fayeez B Tuani Ibrahim, a Masters holder in Electronics \& Telecommunication, is a vivid computer networking enthusiast. His specific research interest includes low power network design and programming. $\mathrm{He}$ is a certified Cisco Network Analyst.

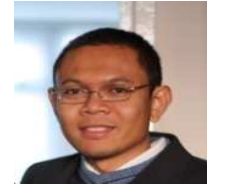

Dr. Zahriladha Zakaria, PhD, MIEEE, BEM, Grad IEM is currently working as a lecturer at the Faculty of Electronic and Computer Engineering, University Teknikal Malaysia Melaka. (UTeM). His research interests include a variety of microwave device development such as planar and non-planar microwave filters, amplifiers and antennas.

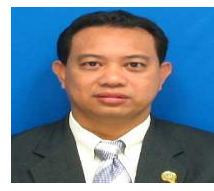

Associate Professor Dr. Abdul Rani Othman , $\mathrm{PhD}$, is currently working as lecturer at the Faculty of Electronic and Computer Engineering, University Teknikal Malaysia Melaka. (UTeM). His research interests include a variety of microwave device development, radio wave propagation, amplifiers and antennas.

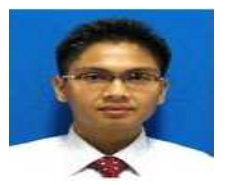

Anas Abdul Latiff received the B.Eng in electrical from Universiti Tun Hussein Onn, Malaysia, and the M.Eng degree in telecommunication from University of Malaya, Kuala Lumpur, Malaysia, in 2009 and 2012, respectively. He is currently a Lecturer with the Department of Telecommunication Engineering, Universiti Teknikal Malaysia Melaka. His current research interests include electronics, photonics and fiber optic device. 